\title{
Adaboost Based ECG Signal Quality Evaluation
}

\author{
Zeyang Zhu' ${ }^{1}$, Wenyang Liu', Yang Yao ${ }^{1}$, Xuewei Chen ${ }^{1}$, Yingxian Sun ${ }^{2}$, Lisheng $\mathrm{Xu}^{1,3^{*}}$ \\ ${ }^{1}$ Sino-Dutch Biomedical and Information Engineering School, Northeastern University, Shenyang \\ City, Liaoning Province, 110819 China \\ ${ }^{2}$ The First Hospital of China Medical University, Shenyang City, Liaoning Province, China \\ 110122 \\ ${ }^{3}$ Neusoft Research of Intelligent Healthcare Technology, Co. Ltd, Shenyang 110167, China
}

\begin{abstract}
Cardiovascular disease is one of the major diseases that threaten human health. Electrocardiogram (ECG) signal is an important indicator for the diagnosis of cardiovascular disease. Accurate analysis of ECG plays a key role in the diagnosis of cardiovascular disease. Underdeveloped areas have always been a high-risk area for cardiovascular disease and there are few doctors for diagnosing cardiovascular disease. One solution is using a telemedicine system for disease diagnosis. However, the quality of the ECG signal collected is not necessarily reliable and may impact diagnosis. In order to solve the problem, we have studied various methods for assessing the quality of ECG signals. In the paper, we analyzed the 12-lead ECG data provided by PhysioNet and selected two features of the time domain: the number of $R$ peaks and the amplitude difference. These two features were extracted from the ECG data to form a matrix of 24 features. We trained the classification model with the feature matrix and achieved a classification accuracy of $95.80 \%$ on the test set. Experimental results demonstrated that the proposed Adaboost algorithm had advantages in accuracy compared with other algorithms for solving ECG quality assessment problems.
\end{abstract}

\section{Introduction}

Cardiovascular disease is the leading cause of death in world according to the World Health Organization [1]. Electrocardiogram (ECG) signal is the most effective monitoring indicator for cardiovascular disease. However, the amplitude of ECG signal only ranges from $0.1 \mathrm{mV}$ to $5 \mathrm{mV}$ [2] and there are various disturbances in the acquisition of ECG signals [3]. Some common noise disturbances of ECG signals such as motion artifact may lead to a particularly poor signal quality. Therefore, the quality of ECG needs to be assessed.

According to the survey [5], the mortality rate of cardiovascular disease in rural areas has been much higher than in cities. Furthermore, cardiovascular disease mortality rates in poor countries and regions are also higher than in rich countries and regions. In order to solve the problem, most of these areas are equipped with some portable ECG devices to provide ECG detection for residents. Some systems that provide telemedicine services have also been developed to address these conditions. With telemedicine systems, users can send their ECG data to cardiovascular experts all around the world to diagnose their condition in real time. However, due to the lack of professional technicians to operate ECG equipment, a potential problem is quality control of the collected signal [4]. If the poor quality signal is submitted to the doctor for diagnosis, it may cause the doctor to misdiagnose and waste the medical resources. In order to solve the problem, many researchers have developed various quality assessment techniques and have achieved high accuracy rate in the classification of ECG quality. In this paper, we tried to improve the accuracy on ECG quality assessment. Adaboost combines multiple weak classifiers into a strong classifier and more accurate classifiers will classify more data. Therefore, Adaboost has a high accuracy in dealing with classification problems. ECG quality assessment is essentially a classification of ECG quality and using adaboost may yield good results. So we used the proposed Adaboost algorithm to access ECG quality and successfully achieved higher accuracy.

\section{Methods}

\subsection{Dataset}

In the paper, the ECG dataset comes from the public available dataset for PhysioNet Computing in Cardiology 
2011. The dataset provides 1500 groups of ECG signal including training and test. The challenge data are standard 12-lead ECG recordings (leads I, II, II, aVR, aVL, aVF, V1, V2, V3, V4, V5 and V6) with full diagnostic bandwidth $(0.05$ through $100 \mathrm{~Hz})$ [5]. Each lead is a 10 second ECG signal with a sampling frequency of $500 \mathrm{~Hz}$ and 16-bit resolution.

\subsection{Classifier algorithm}

In the paper, we proposed a method for binary classification of ECG signal quality using Adaboost algorithm which classifies ECG signals into acceptable ("1") and unacceptable ("-1"). Adaboost algorithm is a kind of boosting and iterative algorithm. The algorithm is one of the most popular ones for classification [6]. The core idea of Adaboost algorithm is to train multiple weak classifiers using the same dataset and then combine these weak classifiers to form a strong classifier. We used the default single-layer decision tree as a weak classifier. Figure 1 illustrates the composition of Adaboost algorithm.

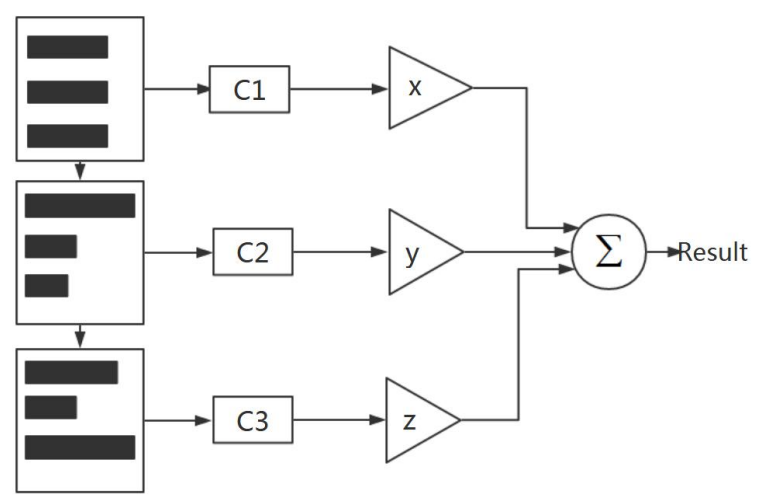

Figure 1. The schematic diagram of the Adaboost algorithm.

Left side of Figure 1 is the data set where the width of the black rectangle represents the different weights of each sample. C represents weak classifiers. $\mathrm{x}, \mathrm{y}, \mathrm{z}$ represent the weights of weak classifiers when they are superimposed.

As shown in Figure 1, give each training sample the same weight of $1 / \mathrm{N}$ ( $\mathrm{N}$ is the number of the samples) when a training data set $\mathrm{T}$ is given as the input $(\mathrm{T}=\{(\mathrm{x} 1$, $\mathrm{y} 1),(\mathrm{x} 2, \mathrm{y} 2) \ldots(\mathrm{xN}, \mathrm{yN})\}, \mathrm{x}$ is the sample and $\mathrm{y}$ is the given label $\{1,-1\})$. The initial weight distribution is given by Equation 1:

$$
D_{1}=\left(w_{1} \ldots w_{1 i} \ldots w_{1 N}\right), w_{1 i}=\frac{1}{N}, i=1,2 \ldots N
$$

The Iteration is according to different weight distributions and the times of iterations is denoted as $\mathrm{m}$, $\mathrm{m}=1,2,3 \ldots \mathrm{M}$. The final strong classifier is derived from the following steps:

1) Train the basic classifiers $C_{m}(x)$ by training data with a weight distribution of $D_{m}$ (weak classifier is a single-layer decision tree).

2) Calculate the classification error rate of $C_{m}(x)$ :

$$
e_{m}=\sum_{i=1}^{N} w_{m i} I\left(C_{m}(x) \neq y_{i}\right)
$$

where function $I$ represents a sample whose statistics are misclassified. $e_{m}$ is the sum of the weights of the misclassified samples.

3) Calculate the coefficient $a_{m}$ of $C_{m}$ to indicate the importance of the weak classifier in the strong classifier:

$$
a_{m}=\frac{1}{2} \log \frac{1-e_{m}}{e_{m}}
$$

4) Redistribute the weight distribution of the sample:

$$
\begin{gathered}
D_{m+1}=\left(w_{m+1,1} \ldots w_{m+, i} \ldots w_{m+1, N}\right) \\
w_{m+1, i}=\frac{w_{m i}}{Z_{m}} \exp \left(-a_{m} y_{i} C_{m}\left(x_{i}\right)\right) \\
Z_{m}=\sum_{i=1}^{N} w_{m i} \exp \left(-a_{m} y_{i} C_{m}\left(x_{i}\right)\right), i=1,2 \ldots, N
\end{gathered}
$$

$Z_{m}$ is to make $D_{m+1}$ a probability distribution.

5) Combine weak classifiers into strong classifier:

$$
C(x)=\operatorname{sign}\left(\sum_{m=1}^{M} a_{m} C_{m}(x)\right)
$$

The derived strong classifier is the integrated learning classifier we need. We put the features extracted from the 12-lead ECG signal into the Adaboost classifier as samples and got the prediction labels.

\subsection{Feature selection}

We selected two features (the number of $\mathrm{R}$ peaks as feature A and the amplitude difference of the ECG signals as feature B) as the data for the sample. In fact, since each lead can extract features with different values, each sample is equivalent to containing 24 features.

1) Number of $R$ peaks:

The $\mathrm{R}$ peaks are the positive peaks of the QRS regions [7] which reflect the size of the RR interval. Figure 2 depicts a typical ECG signal. The RR interval of the ECG signal of normal sinus rhythm ranges from $0.6 \mathrm{~s}$ to $1 \mathrm{~s}$ so the number of R peaks for 10 second of ECG ranges from 10 to 20 . Certain heart diseases such as atrial fibrillation [8] can cause the RR interval to be too long and the number of $\mathrm{R}$ peaks to decrease. However, a high quality ECG signal does not have a large decrease or increase in the number of $\mathrm{R}$ peaks. If this happens, the quality of the ECG signal can be judged as unacceptable. Figure 2 showed a good quality ECG signal with R peaks marked, 
Figure 3 showed a low-quality ECG signal with R peaks marked.

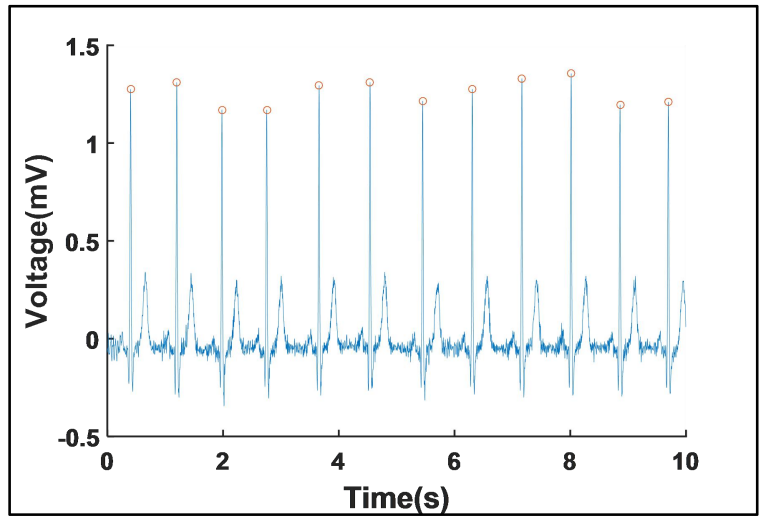

Figure 2. Typical high-quality ECG signal and its corresponding $\mathrm{R}$ peaks detected.

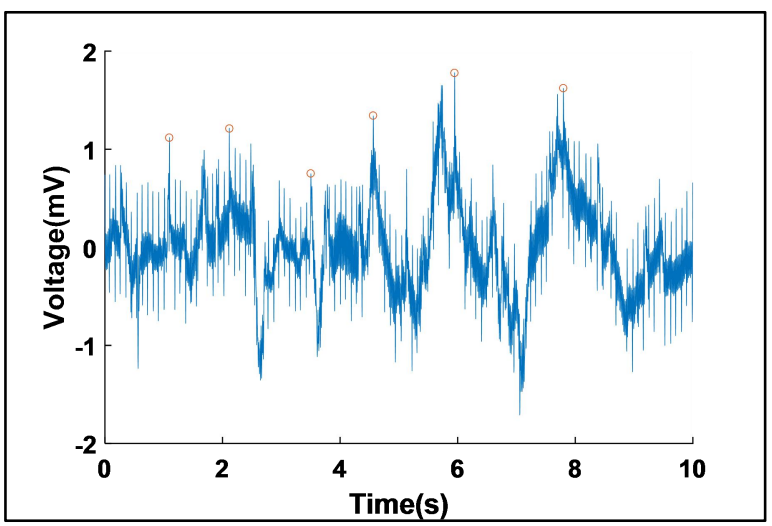

Figure 3. Typical low-quality ECG signal and its corresponding $\mathrm{R}$ peaks detected.

\section{2) Amplitude difference of the ECG:}

The difference in the amplitude value of the ECG signal is used to determine whether the lead is off during the signal acquisition process. The lead-off performance is characterized by a long-term constant value of the collected ECG signal. If the lead is detached, it means that the ECG record is unacceptable. The difference between the maximum and minimum values of the ECG signal amplitude reflects the lead-off condition, which we use as another characteristic indicator.

Differential threshold method [9] was used to find $\mathrm{R}$ peaks. Feature B was obtained from the difference between the maximum and minimum values of each ECG records. Then we spliced the values of all the features in a certain order. The rule is: 12 features of 12 leads per line and each column is a feature of all ECG signal. This constituted the feature matrices of the two kinds of features and finally the two matrices were spliced into one matrix as the sample.

Our approach is based on the Windows platform and the results are from MATLAB R2016a. The operating environment of the algorithm is: 64-bit Windows7
Ultimate, PC CPU is Intel(R) Core(TM) i5-4210M, 2.60GHz, 8G RAM.

\section{Results}

\subsection{Evaluation Index}

In our research, we used sensitivity (true negative rate) and specificity (true positive rate) combined with accuracy to measure the performance of ECG signal classification. Sensitivity and specificity are defined by Equations 6 and 7.

$$
\begin{aligned}
& \text { Sensitivity }=\frac{T P}{T P+F N} \\
& \text { Specificity }=\frac{T N}{T N+F P}
\end{aligned}
$$

TP (True Positive) represents the number of the acceptable ECG records be correctly classified as acceptable. TN (True Negative) represents the number of the unacceptable ECG records be correctly classified as unacceptable. FP (False Positive) represents the number of the unacceptable ECG records be classified as acceptable. FN (False Negative) represents the number of the acceptable ECG records be classified as unacceptable.

\subsection{Performance of classifier algorithm}

We used 1000 ECG recordings for training and 500 sets of ECG signal data for testing. The performance of the proposed algorithm applied to ECG quality assessment was summarized in Table 1 .

Table 1. Sensitivity, specificity and accuracy achieved for training set and testing set of the PhysioNet CinC 2011 dataset.

\begin{tabular}{ccc}
\hline Index & Training set & Testing set \\
\hline TP (number) & 772 & 370 \\
TN (number) & 206 & 109 \\
FP (number) & 20 & 16 \\
FN (number) & 2 & 5 \\
Sensitivity(\%) & 99.7 & 98.6 \\
Specificity(\%) & 91.5 & 87.2 \\
Accuracy(\%) & 97.8 & 95.8 \\
\hline
\end{tabular}

As shown in Table 1, the proposed Adaboost algorithm correctly classified 978 sets of signals on the training set, yielding a classification accuracy of $97.8 \%$. On the testing set, Adaboost correctly classified 370 recordings as acceptable and 109 recordings as unacceptable, yielding a classification accuracy of $95.8 \%$. On the testing set, the sensitivity was $98.6 \%$ and the specificity 
was $87.2 \%$.

We had reviewed and compared the research on the quality assessment of ECG from the same dataset (PhysioNet) by other researchers in recent years. The results of the comparison were summarized in Table 2.

Table 2. Comparison with other existing ECG signal quality classification approaches (Accuracy on PhysioNet CinC 2011 dataset).

\begin{tabular}{cc}
\hline Classification algorithm & Accuracy(\%) \\
\hline Adaboost & $\mathbf{9 5 . 8}$ \\
QRS detection & 93.4 \\
LS-SVM & 92.2 \\
KSVM & 92.0 \\
Raf & 91.0 \\
ORaf & 92.0 \\
Multi-feature Fusion & 91.6 \\
\hline
\end{tabular}

The accuracy of the six methods was given in table 2 . A research used QRS wave detection to evaluate ECG signal quality and the accuracy rate reached $93.4 \%$ [10]. A paper compared the accuracy of LS-SCM, KSVM, RaF and $\mathrm{ORaF}$ for ECG signal quality classification [11]. The paper point out that using LS-SVM for classification can get the highest accuracy of $92.20 \%$, and the corresponding results of the other three classifiers were $92.0 \%, 91.0 \%, 92.0 \%$. A research used a multi-feature fusion method to evaluate ECG signal quality with a classification accuracy of $91.6 \%$ [12]. In general, Adaboost has certain advantages in accuracy.

\section{Conclusion}

Portable ECG signal acquisition equipment provides protection for cardiovascular health monitoring. It also brings the problem that non-professionals cannot recognize the quality of ECG signals. The research is dedicated to helping non-professional users confirm the quality of ECG signal and reduce the burden on cardiovascular disease specialists in hospitals. What's more, the research committed to improve the accuracy of ECG quality assessment.

In this paper, a method for classifying the quality of ECG signal using Adaboost algorithm was proposed and higher classification accuracy was obtained. However, the specificity on the test set was only $87.2 \%$ which indicated the request for improvement in our work. In the future, we will extract more kinds of features from ECG signal and increase the number of features in the sample which is expected to improve the specificity of classification.

\section{Acknowledgments}

The research reported here was, in part, supported by the National Natural Science Foundation of China (No. 61773110, No. 61374015), and the Fundamental Research Funds for the Central Universities (No. N161904002, No. N172008008). This work is also supported by the Open Program of Neusoft Research of Intelligent Healthcare Technology, Co. Ltd, Item number NRIHTOP1801.

\section{References}

[1] Johannesen L, Galeotti L, "Automatic ECG quality scoring methodology: mimicking human annotators," Physiological Measurement, vol. 354, no. 9, pp. 1479-1489, Feb. 2012.

[2] Ma J, Xu J, Xu H B, et al. "Design of ECG signal acquisition and processing circuit," Applied Mechanics and Materials, vol.236-237, no. 1, pp. 856-861, Nov. 2012.

[3] Li, Liping. "A quality assessment method of single-lead ECG signal based on spectral analysis," International Conference on Information Technology in Medicine \& Education IEEE, July. 2017.

[4] Xia, Henian, et al. "Computer algorithms for evaluating the quality of ECGs in real time.," Computing in Cardiology, Mar. 2011.

[5] http://www.physionet.org/challenge/2011/

[6] Liu, Xiangyang, et al. "A preprocessing method of AdaBoost for mislabeled data classification," 2017 29th Chinese Control And Decision Conference (CCDC) IEEE, July. 2017.

[7] Sadhukhan, Deboleena, and M. Mitra. "R-Peak detection algorithm for Ecg using double difference and RR interval processing," Procedia Technology, vol. 4, no. 1, pp. 873877, Feb. 2012.

[8] Matsui, Taishi, et al. "Analysis of variability of R-R intervals for the diagnosis of atrial fibrillation: A new algorithm," Journal of Electrocardiology, vol. 51, no. 20, pp. 382-385, May-June. 2018.

[9] Lai, Dakun, F. Zhang, and C. Wang. "A real-time QRS complex detection algorithm based on differential threshold method," 2015 IEEE International Conference on Digital Signal Processing (DSP) IEEE, Sept. 2015.

[10] Hayn, Dieter, B. Jammerbund, and Schreier, Günter. "QRS detection based ECG quality assessment," Physiological Measurement, vol. 33, no. 9, pp. 1449-1461, Aug. 2012.

[11] Zhang, Yatao, et al. "Comparing the performance of Random Forest, SVM and their variants for ECG quality assessment combined with nonlinear features," Journal of Medical and Biological Engineering, vol. 4, no. 4, pp. 1-12, Apr. 2018.

[12] Yong Xia, Honghong Jia. "ECG quality assessment based on multi-feature fusion," International Conference on Natural Computation, Fuzzy Systems and Knowledge Discovery. IEEE, June. 2017.

Address for correspondence:

Lisheng $\mathrm{Xu}$.

Northeastern University, No. 195, Chuangxin Road, Hunnan District, Shen Yang City, Liaoning Province, China.

xuls@bmie.neu.edu.cn. 